Audiology

\title{
Genes important for otoneurological diagnostic purposes - current status and future prospects
}

\author{
Geni importanti per la diagnostica otoneurologica: stato dell'arte e prospettive future
}

K. PAWLAK-OSIŃSKA', K. LINKOWSKA², T. GRZYBOWSKI²

${ }^{1}$ Department of Otolaryngology and Oncology Collegium Medicum in Bydgoszcz Nicolaus Copernicus University, Skłodowskiej-Curie 9, Bydgoszcz, Poland; ${ }^{2}$ Department of Forensic Medicine Division of Molecular and Forensic Genetics Collegium Medicum in Bydgoszcz Nicolaus Copernicus University, Skłodowskiej-Curie 9, Bydgoszcz, Poland

\begin{abstract}
SUMMARY
This review focuses on the current knowledge of the genes responsible for non-syndromic hearing loss that can be useful for otoneurological diagnostic purposes. From among a large number of genes that have been associated with non-syndromic hearing impairment, we selected several best-known genes, including the COCH gene, GJB2, GJB6 and SLC26A4, and we describe their role and effects of mutations and prevalence of mutations in various populations. Next, we focus on genes associated with tinnitus. Important areas for further research include assessment of genes potentially involved in pathophysiology of tinnitus and vertigo, which have traditionally been considered as being of otological aetiology, while advances in neuroimaging techniques have increasingly shifted studies toward neurological correlations.
\end{abstract}

KEY WORDS: Genetics • Tinnitus • Vertigo $\bullet$ Hearing impairment $\bullet$ Epilepsy

\section{RIASSUNTO}

La presente review tratta lo stato dell'arte nella conoscenza dei geni responsabili delle ipoacusie non sindromiche, che potrebbero risultare utili a scopo diagnostico nell'ambito della otoneurologia. Tra i diversi geni identificati in associazione ad un calo dell'udito di tipo non sindromico, ne sono stati selezionati alcuni tra i più noti, come il gene COCH, GJB2, GJB6 e SLC26A4. Di questi vengono descritti il ruolo, l'effetto delle mutazioni a carico e la prevalenza delle stesse mutazioni in diverse popolazioni. Successivamente ci si focalizza sui geni associati al tinnito. Una valida area di ricerca per il futuro è infatti data dall'identificazione di geni potenzialmente coinvolti nella fisiopatologia del tinnito e della vertigine, condizioni tradizionalmente considerate di eziologia otologica, ma che, a seguito di un avanzamento delle tecniche di neuroimaging, si è sempre più propensi a correlare con la sfera neurologica.

PAROLE CHIAVE: Genetica $\bullet$ Tinnito $\bullet$ Vertigine $\bullet$ Ipoacusia $\bullet$ Epilessia

Acta Otorhinolaryngol Ital 2018;38:242-250

\section{Introduction}

The inner ear consists of two organs that are evolutionarily related and have a similar physiology: the cochlea and the peripheral vestibular system (labyrinth). The cochlea is involved in sound perception. The vestibular labirynth consists of the sacculus and utriculus, which predominantly register linear accelerations, including gravity, and the semicircular canals, which register rotary motions ${ }^{1}$. Due to the remarkable resemblance between both parts of the inner ear, it seems logical that a number of inner ear-specific genes will have both cochlear and vestibular functions, and consequently, mutations in these genes would be expected to lead to both auditory and vestibular dysfunction. Of the many described in the literature, we selected genes which may be the starting point for rapid otoneurological diagnosis. We considered both the impact of mutation on phenotype and differences in the frequency of mutations between populations. For brevity, proposed genes are summarised in Table I. In the first part of this review, we focus on the genes considered responsible for non-syndromic hearing loss that can be useful for otoneurological diagnostic purposes. Next, we discuss the genes potentially involved in the pathophysiology of vertigo and tinnitus, which have traditionally been considered as being of otological aetiology, while advances in neuroimaging techniques have increasingly shifted studies toward its neurological correlation. 
Table I. Genes proposed for otoneurological diagnostic purposes.

\begin{tabular}{|c|c|c|c|}
\hline Gene & Gene function & Disorder & References \\
\hline $\mathrm{COCH}$ & Cochlin & $\begin{array}{l}\text { Non-syndromic hearing loss } \\
\text { with/without vestibular dysfunction }\end{array}$ & $\begin{array}{l}\text { Chen et al., } 2013 \\
\text { Kim et al., } 2016 \\
\text { Usami et al., } 2003 \\
\text { Fransen et al., } 2001 \\
\text { Kamarinos et al., } 2001 \\
\text { Collin et al., } 2006 \\
\text { de Kok et al., } 1999 \\
\text { Street et al., } 2005 \\
\text { Gallant et al., } 2013\end{array}$ \\
\hline GJB2 & Gap junction protein beta-2 & Non-syndromic hearing loss & Zheng et al., 2015 \\
\hline GJB6 & Gap junction protein beta- 6 & Non-syndromic hearing impairment & $\begin{array}{l}\text { del Castillo et al., } 2002 \\
\text { Seeman et al., } 2005\end{array}$ \\
\hline SLC26A4 & Pendrin & $\begin{array}{l}\text { Pendred syndrome, non-syndromic } \\
\text { hearing loss with/without EVA }\end{array}$ & $\begin{array}{c}\text { Miyagawa et al., } 2014 \\
\text { Tsukada et al., } 2015 \\
\text { Yang et al., } 2009\end{array}$ \\
\hline KCNE1 & $\begin{array}{c}\text { Potassium voltage-gated channel subfamily } \mathrm{E} \\
\text { member } 1\end{array}$ & Chronic tinnitus & $\begin{array}{l}\text { Sand et al., } 2010 \\
\text { Pawełczyk et al., } 2012\end{array}$ \\
\hline KCNE3 & $\begin{array}{c}\text { Potassium voltage-gated channel subfamily } \mathrm{E} \\
\text { member } 3\end{array}$ & Chronic tinnitus & Sand et al., 2011 \\
\hline KCNQ4 & $\begin{array}{l}\text { Potassium voltage-gated channel subfamily } Q \\
\text { member } 4\end{array}$ & Non-syndromic hearing loss & $\begin{array}{l}\text { Kim et al., } 2011 \\
\text { Uehara et al., } 2015\end{array}$ \\
\hline KCTD12 & $\begin{array}{l}\text { Potassium channel tetramerisation domain } \\
\text { containing } 12\end{array}$ & Chronic tinnitus & Sand et al., 2012a \\
\hline GDNF & Glial cel derived neurotrophic factor & Chronic tinnitus & Sand et al., 2012b \\
\hline BDNF & Brain derived neurotrophic factor & Chronic tinnitus & Sand et al., 2012b \\
\hline
\end{tabular}

Genes associated with hereditary hearing loss

Hearing impairment is the most common human communication disorder and hereditary causes play an important role in its aetiology. Genetic hearing loss can be classified into non-syndromic and syndromic hearing loss. Nonsyndromic hearing loss (NSHL) constitutes approximately $75 \%$ of cases of genetic hearing loss. To date, more than 90 genes and 140 loci have been associated with non-syndromic hearing impairment (Hereditary Hearing Loss homepage, http://hereditaryhearingloss.org/main. aspx?c=.HHH\&n=86162). Non-syndromic deafness can be autosomal dominant (DFNA), autosomal recessive (DFNB), or X-linked (DFNX). Hearing loss can also be caused by mutations in mitochondrial DNA (mtDNA) ${ }^{2}$. Balance problems are also relatively frequent, but considerably less is known about the causes. However, it is commonly known that many hearing-impaired people also suffer from vertigo. Moreover, it is now recognised that many syndromes with genetic hearing impairment also show dysfunction of the vestibular system ${ }^{3}$.

\section{$\mathrm{COCH}$}

One of the most common forms of DNFA hearing loss, which is clinically characterised by late onset progressive sensorineural hearing loss accompanied by vestibular dysfunction, are mutations at the DFNA9 locus, localised to chromosome 14q12-q13. Mutations at this locus result in alterations in the cochlin protein, encoded by the $\mathrm{COCH}$ gene ${ }^{4}$. Cochlin is a 550-amino acid extracellular protein that consists of a signal peptide, LCCL domain and two vWFA domains, and has been reported to be expressed most abundantly in the inner ear ${ }^{5}$. Mutated forms of cochlin have been found to interfere with disulphide bonds and correct protein folding, resulting in disturbances affecting substantial cochlear functions ${ }^{6}$. The first three nucleotide changes cause missense mutations in $\mathrm{COCH}$, resulting in amino acid substitutions of Val to Gly at codon 66 (p.V66G), Gly to Glu at codon 88 (p.G88E) and Trp to Arg at codon 117 (p.W117R) were found in hearingimpaired family by Robertson et al. ${ }^{7}$. These residues are localised in LCCL domain, in close proximity to a cluster of cysteines, near the amino terminus of $\mathrm{COCH}^{7}$. The mutation p.Ala119Thr located in close spatial proximity to $\mathrm{W} 117$ was found to be responsible for autosomal dominantly inherited hearing loss accompanied by vestibular symptoms in a Japanese population ${ }^{8}$. The substitution of conserved proline for a serine at position 51 of the protein (p.P51S) has been found in Dutch, Belgium and The 
Netherlands DFNA9 families ${ }^{19}$. Kamarinos et al. found a mutation changing the isoleucine 109 to an asparagine (I109N) in a family with DFNA9 deafness and vestibular disorder from Australia ${ }^{10}$. The highly conserved amino acid glycine at codon 87 was found to be changed as two separate mutations, p.G87W in the Dutch DFNA9 family and p.G87V in a Chinese DFNA9 family ${ }^{511}$. The first mutations found outside of the LCCL domain was p.C542F in exon 12 , which is in the von Willbrand factor A2 (vWFA2) domain ${ }^{12}$. Another mutation in vWFA2 domain is 18 base pair deletion, resulting in an in-frame deletion of 6 amino acids, p.Ile399_Ala404del found in family with sensorineural hearing impairment and tinnitus ${ }^{13}$. The mutations in $\mathrm{COCH}$ gene are a frequent cause of late onset hearing impairment, and would imply $\mathrm{COCH}$ as a major gene for cochleovestibular impairment. Coch-/- mice do not develop the degeneration and hearing loss as observed in DFNA9 patients, suggesting that DFNA9-associated $\mathrm{COCH}$ mutations may be gain of function in nature ${ }^{14}$. Yao et al. proposed that misfolding of mutant and WT cochlins in dimers and oligomers leads to a toxic gain of function that is responsible for the cellular degeneration and hearing loss in DFNA9 patients ${ }^{6}$. Recently, a genotype-phenotype correlation was proposed that individuals with vWFA domain mutations predominantly exhibit hearing loss, while individuals with LCCL domain mutations have hearing loss accompanied by vestibular dysfunction ${ }^{15} 16$.

\section{GJB2}

The GJB2 gene encodes a protein called connexin 26 (Cx26), which is member of the connexin protein family. Connexin proteins form channels called gap junctions, which allow communication between adjacent cells. Unlike typical ion channels, gap junction channels possess a relatively large pore size and can allow passage of ions, cell signaling molecules and small molecules up to $\sim 1.5 \mathrm{kDa}$. This direct intercellular communication pathway plays an important role in embryonic and postembryonic development, cancer suppression and many physiological and pathological functions. Connexin mutations can cause severe deafness, indicating that connexin gap junctions play a critical role in hearing. The pathogenesis and deafness mechanisms underlying connexin mutation-induced hearing loss - a common hereditary deafness have been extensively summarised by previous reviews e.g. ${ }^{17}-$. More than 150 different GJB2 variants have been identified, including missense, nonsense and frameshift mutation (the Connexin-deafness homepage, http://davinci. crg.es/deafness). Moreover, the spectrum of GJB2 mutations vary among different ethnic groups. For example, the GJB2 c.35delG mutation is the most common cause of hearing loss worldwide and is identified frequently in European, North African, Middle East Asian and American populations, but rarely in South Asian, Southeast Asian, or East Asian countries. The GJB2 c.235delC mutation is one of the most common genetic causes of hearing loss in East Asian countries (Japan, Korea, and China). In South Asian countries (India, Pakistan, and Bangladesh) the most common cause of hearing loss is GJB2 p.W24X mutation, which is also found in Middle Eastern and East European populations at a moderate frequency and at a low frequency in Southeast Asian populations, but it was not observed in African or East Asian populations. The GJB2 p.V37I mutation is the most common cause of hearing loss in Southeast Asian and Oceanic countries (Thailand, Malaysia, Indonesia and Taiwan). The GJB2 c.167delT mutation is the most frequent variant in Ashkenazi Jewish families ${ }^{18} 19$. Thus, knowledge of ethnic and regional differences in the GJB2 mutation spectrum is very important and could help guide genetic testing and assist in clinical decision making.

\section{GJB6}

In addition to GJB2 (Cx26), multiple connexin genes, GJB6 (Cx30), GJB3 (Cx31), GJC3 (Cx29) and GJA1 (Cx43), have been identified in the cochlea, but compared to the extensive expression of Cx26 and Cx30, the expression of other connexins in the cochlea is limited. Mutations of these connexin genes can also cause hearing loss. Del Castillo et al. (2002) identified a 342-kb deletion in the GJB6 gene which is the second most frequent (after the 35delG mutation in GJB2) genetic cause of nonsyndromic prelingual hearing impairment in the Spanish population ${ }^{20}$. A multicentre study, involving 9 countries, showed that the GJB6 deletion was present in most of the screening populations, with higher frequencies in France, Spain and Israel ${ }^{21}$. In the Czech population, the $342-\mathrm{kb}$ deletion in the GJB6 gene was identified in 1 of 13 patients with prelingual nonsyndromic sensorineural deafness who also carried 1 pathogenic mutation in the GJB2 gene and was not detected in 600 control chromosomes from individuals with normal hearing ${ }^{22}$. Seeman et al. (2005) concluded that the $342-\mathrm{kb}$ deletion is very rare in central Europe compared to reports from Spain, France and Israel.

In contrast to the results of European and Asian populations mutations, neither GJB2 nor GJB6 are associated with non-syndromic deafness in Africans ${ }^{23-25}$. The data indicate that the high frequency of GJB2 and GJB6 mutations in non-syndromic hearing loss have evolved in Eurasian populations after their migration out of Africa, and 
spread with population migrations due to founder effects, and not to a putative "protective" variant in the genomic structure in Africans ${ }^{26}$. Therefore, both GJB2 and GJB6 should not be investigated routinely in clinical testing in patients of African ancestry.

\section{$S L C 26 A 4$}

The SLC26A4 mutations are the second genetic cause of NSHL after GJB2 mutations. The SLC26A4 gene, encodes a transmembrane protein (pendrin) expressed in thyroid gland, inner ear and kidney, where it is involved in the transport of anions such as iodide, chloride and bicarbonate. Mutations in the SLC26A4 gene are known to be responsible for a broad phenotypic spectrum, from typical Pendred syndrome, characterised by hearing loss, goiter and eventually hypothyroidism, with/without enlarged vestibular aqueduct (EVA) or other inner ear malformations to non-syndromic hearing loss, called DFNB4, characterised by hearing loss with/without EVA ${ }^{27}$. The prevalent of SLC26A4 mutations (90\% in Pendred syndrome and $78.1 \%$ in non-syndromic hearing loss associated with EVA) indicates the importance of this gene in the pathophysiology of this category of hearing impairment ${ }^{28}$. More than 170 mutations have been found in SLC26A4 gene (Pendred/BOR homepage, http://www.healthcare. uiowa.edu/labs/pendredandbor/), and as in the case of GJB2 different mutational spectrums among different ethnic groups have been reported. Studies on NSHL have revealed biallelic SLC26A4 mutations in $2 \%$ to $3.5 \%$ of Caucasian patients, but in $5.5 \%$ to $12.6 \%$ of East Asian patients. Moreover, the most common SLC26A4 mutations found in the Asian population were quite different from that found in the Caucasian population. The most common mutations of SLC26A4 gene observed in the East Asian, South Asian and Southeast Asian populations were p.H723R, c.919-2A $>$ G and p.V239D. These mutations were not detected in the Caucasian population. The c.1001+1G>A, p.V138F, p.T416P, p.L236P and p.G209V mutations are prevalent in the Caucasian population ${ }^{18}$.

\section{Genetics of vestibular disorders}

The most representative otoneurological vestibular disease located in the labyrinth is Meniere's disease. Numerous hypotheses have attempted to explain the aetiology of the disease. However, the anamnesis usually confirms the familiar history of Meniere's disease. A genetic basis, involving DTNA and FAM136A genes, is believed to be responsible for familiar incidents of the disease ${ }^{29}$. The situation is complicated because Meniere's disease is variously expressed through pronounced cochlear or dominated vestibular syndromes. Finally, 83 genes are sus- pected to be altered when balance and hearing symptoms are present. Some of them, such as CRYM and OTOG, are more specific for the vestibule, while others, such as AQP1, COL11A2 and OTOS, are expressed much higher in the cochlea; MYO6 and USH1C are associated with both hearing and vestibular defects ${ }^{30}$. Li et al. reported on 15 genes that are strongly inferred with Meniere's disease. The most important are CD4, NOTCH2 and IL6 ${ }^{31}$. Eppsteiner and Smith suggested a special phenotype for Meniere's patients associated with DFNB1 ${ }^{32}$. Looking at a biochemical cause of Meniere's disease, a hypothesis of disturbed ionic transport following rs 3746951 and rs487119 mutations has been taken into consideration ${ }^{33}$.

The most common vestibular disease according to otoneurological literature is benign paroxysmal positional vertigo (BPPV). Its aetiology is correlated with head injury and sense organ degeneration in an advanced age. The only treatment is canalith repositioning procedure, but its success is varies individually. Tsai at al. reported on the influence of miR-34a and Sirtuin 1 (SIRT1) gene expression on the effect of therapy ${ }^{34}$. Canalith repositioning decreased PPAR- $\gamma$, PGC- 1 and FoxO gene expressions just after the procedure. After complete cure of BPPV, the previously decreased expression increased together with he ROS concentrations, pro-inflammatory cytokine concentrations and p53 levels ${ }^{34}$.

Apart from vertigo originating from the labyrinth, the central nervous system disorders are followed by dizziness or vertigo. The migraine represents them. Vestibular migraine is clinically difficult to confirm - it is not clear if it is real vestibular migraine or if vestibular symptoms belong to aura of regular migraine -. Nevertheless, the family penetrance and interaction of genes are observed in vestibular migraine or vestibular component of typical migraine. Until now, no specific gene has been identified. Because migraine can be associated with ataxia, the GBA2, TGM6, ANO10, SYT14, KCNA1 and CACNA1A genes are destined as possible targets ${ }^{35}$. Typical vestibular migraine as a subtype of migraine seems to be connected with GWAS and endophenotypes of MTDH, TRPM8 and LRP1 genes ${ }^{36}$.

Up to the present moment, numerous dizziness and vertigo patients are still not identified as suffering from special vestibular disease. For these idiopathic cases, the name of "recurrent vertigo" is accepted. For the future genetic investigations, this group is the most interesting. Initially, the regions $22 \mathrm{q} 12$ and $5 \mathrm{p} 15$ are designated as potentially responsible for vestibular pathology ${ }^{37}$.

\section{Tinnitus associated genes}

Tinnitus, which affects approximately 40 million people worldwide chronically, is currently the biggest challenge 
of otoneurological diagnostics. It is believed that every individual over 20 has experienced tinnitus at least once. The aetiology of tinnitus, defined as hearing sounds with no audible sources in the environment is unknown, but an established association with various forms of sensorineural hearing impairment and frequent precipitation by noise exposure suggest substantial overlap with pathologies of the inner ear, and related disorders of auditory information processing. The genetics of tinnitus and data that suggests a complex or multifactorial genetic aetiology has been discussed in recently published reviews ${ }^{38}$.

While the underlying biological mechanisms are still incompletely understood, research into the pharmacological treatment of tinnitus has emphasised the role of cellular ion regulation and transport ${ }^{39}$.

\section{Annel genes}

Voltage-gated ion channels directly control neural transmission of auditory input. In the inner ear, sensory neurons are surrounded by endolymph rich in $\mathrm{KCl}$ and constantly recycle potassium for the generation of endocochlear potentials. KCNE1, a potassium channel subunit gene has been implicated in maturation defects of central vestibular neurons, in Menière's disease, and in noise-induced hearing loss ${ }^{40}$. Sand et al. (2010) screened 201 Caucasian outpatients with a diagnosis of chronic tinnitus for mutations in the KCNE1 gene. Although no significant differences in KCNE1 coding allele frequencies of control subjects and tinnitus patients were found, Sand et al. (2010) hypothesised that primary chronic tinnitus could be a part of the phenotypic spectrum associated with KCNE1 ${ }^{40}$. Pawełczyk et al. (2012) found that mutations in the KCNE1 gene contributed to tinnitus that developed independently of hearing loss in a Polish population ${ }^{41}$. A prominent role of the KCNE1 subunit in auditory perception is underscored by degeneration of sensory hair cells and deafness in KCNE1 knock-out animals ${ }^{42}$, and deleterious effects of a spontaneous KCNE1 null mutation on hearing in mice ${ }^{43}$. Like KCNE1, KCNE3 is expressed in the mammalian inner ear and brain. Both proteins interact to regulate trafficking, surface expression and activation of another potassium channel, $\mathrm{KCNH} 3$, in the cortex and in other parts of the central nervous system. So far, a limited number of investigations has addressed sequence variations in KCNE3, a linkage hotspot for autosomal recessive, non-syndromic hearing impairment. In an association study involving 288 individuals, no significant effect of KCNE3 on the risk for developing chronic tinnitus or predicting the severity of tinnitus was noted. It is likely that mutations in KCNE3 alone may not be indicative of tinnitus, but when mutations are present in either KCNE3 or the channel with which it is interacting (e.g. KCNQ1) the biophysical properties of the channel complex are significantly altered ${ }^{39}$. An example of coexistence are mutations in other $\mathrm{K}+$ channel gene, KCNJ10 that are associated with non-syndromic hearing loss in carriers of SLC26A4 mutations with an EVA/PS phenotype ${ }^{44}$. Dysfunctional channels and mutations in the gene encoding the KCNQ4 subunit are a hallmark of autosomal dominant deafness $2 \mathrm{~A}^{45}$. KCNQ4 is expressed in the outer sensory hair cells of the cochlea and is responsible for recycling $\mathrm{K}+$ ions after stimulation of the hair cel ${ }^{46}$. KNCQ4 mutations were identified in European, American, Japanese, Taiwanese and Brazilian families with non-syndromic hearing loss ${ }^{47} 48$. So far, no association has been found between mutations in KNCQ4 gene and tinnitus.

A well-established theoretical framework for tinnitus has been provided by the disruption of GABAB receptor signaling in animal models ${ }^{49}$. A key role for GABAB receptors has been confirmed by the effects of receptor agonists on tinnitus symptomatology. An auxiliary subunit that is closely associated with the carboxy terminus of GABAB2 receptors is KCTD12, a potassium channel tetramerisation domain-containing protein. Co-assembly of KCTD12 and GABAB2 changes the properties of the GABAB ${ }^{12}$ core receptor by increasing agonist potency, by altering G-protein signaling, and by promoting desensitisation ${ }^{50}$. A study involving 95 Caucasian outpatients with a diagnosis of chronic tinnitus screened for mutations in the KCTD12 gene revealed weak association with tinnitus but KCTD12 genotype did not predict tinnitus severity ${ }^{51}$.

\section{GDNF and BDNF}

Glial cell-derived neurotrophic factor (GDNF) and brainderived neurotrophic factor (BDNF) play key roles in the early development of the central auditory pathway and the inner ear. Both have been successfully employed to treat experimental forms of hearing loss and are likely to operate in a broad spectrum of auditory phenotypes, including phantom perceptions of sound. Although previous research has suggested a role of variant BDNF in modulating the genetic susceptibility to chronic tinnitus with hearing impairment ${ }^{52}$, a later study failed to observe any major effects of individual BDNF or GDNF variants on susceptibility to the phenotype ${ }^{53}$. The findings are compatible with minor, additive effects, which would appear to be gender-sensitive according to subjective ratings of symptoms, offering a possible explanation for distinct patterns of central nervous activity in tinnitus ${ }^{53}$. 


\section{Epilepsy related genes}

Recent study has found that patients suffering from tinnitus have abnormal electrical activity of the cerebral cortex ${ }^{54} 55$. The presence of abnormal electrical activity of the cerebral cortex in patients with tinnitus, where an important role seems to be played by hyperactivity and epilepsy related activity, implies the assumption that tinnitus can be a substitute for epilepsy. Although the aetiology of epilepsy is not yet fully recognised, it is known that genetic factors play an important role. Research on families suffering from epilepsy concerning a mendelian inheritance mechanism have shown that the genetic basis consists mainly of mutations in genes encoding subunits of ion channels and receptors for neurotransmitters. They do not, however, explain all cases. An example can be generalised epilepsy with febrile seizure (GEFs + ), which essentially is a set of symptoms of epilepsy with different phenotypes. In a family with GEFs + mutations have been identified in the genes encoding subunits of sodium channels (SCN1B, SCN2 and SCN1A) and GABA receptor subunit (GABRG2 and GABRD), but in general these mutations are responsible for only about $10 \%$ of GEFs $+{ }^{56}$. In rare cases, association studies indicated a significant effect of mutations in the genes encoding subunits of the GABA receptor (GABRG2 and GABRA1) and chloride (CLCN2) and calcium channels (CACNA1H), as well as in the gene encoding EFHC1 calcium-binding protein. Nevertheless, most epilepsy cases are determined by multiple genes and the phenotypic effect is a result of coexistence of many common sequence variants ${ }^{57}$. Advances in genomic technology research made in recent years have created new opportunities to identify genetic risk factors. Recent wide-genome association studies and meta-analyses have identified new loci associated with the risk of epilepsy, both in genes encoding ion channels (e.g. rs6732655, rs12987787 and rs11890028 located in the SCN1A gene), as well as genes unrelated to "channelopathies" (e.g. rs1044352 located in PCDH7 gene or rs1939012 located in MMP8 gene) ${ }^{5859}$. The detection of the links between epilepsy and tinnitus is important to the selection of effective treatment. A recent study has showed that disorders characterised by neuronal hyperexcitability, such as epilepsy and tinnitus are associated with a reduction in KCNQ2/3 channel activity. This reduction is due to a shift in the voltage dependence of KCNQ2/3 channel activation to more positive voltages (60). In vivo studies demonstrated that anti-epileptic drugs that a shift in the voltage dependence of KCNQ to more negative voltages prevent the development of tinnitus ${ }^{6061}$.

It is alleged that epilepsy may also play a role in generating vestibular pathology ${ }^{62}$. However, "vestibular epilep- sy" as the cause of vertigo is difficult to prove ${ }^{63}$. Family history of epilepsy, previous head injuries and vestibular symptoms intertwine with other epileptiform symptoms that may indicate this specific form of epilepsy ${ }^{62}$. Since there is no specific test confirming the "vestibular epilepsy", it seems that genetic research may give credence to this diagnosis ${ }^{64}$.

There are also many other types of dizziness of which the origin is unclear. Vertigo lasting seconds is often classified as benign paroxysmal positional vertigo. Such recognition seems to be overused, in many cases tests (such as Hallpike's and roll-on) confirming the diagnosis are controversial and the causes of dizziness remain unknown, or at least uncertain. The situation is similar with regard to dizziness that lasts for minutes or hours (without hearing impairment) and often repeats itself. These symptoms require a long diagnostic process involving many specialists. Vestibular hyperactivity observed during caloric stimulation of the labyrinths is sometimes the only pathological vestibular finding, in such case "channelopathy" may be one of or the only suspected cause of dizziness ${ }^{65}$. Vestibular migraine seems to derive from this neuronal conduction pathology ${ }^{66}$.

\section{Future prospects}

The clinical heterogeneity of tinnitus and its associated comorbidities makes the selection of patients for genomic studies challenging. Improving the diagnosis of tinnitus patients and their categorisation into a homogenous group is essential to investigate the contribution of genetic factors to the development of the disease. New possibilities for better understanding of the tinnitus are provided by TINNET, a European research network created in 2014 (http://tinnet.tinnitusresearch.net/index.php). The strategy of TINNET is to standardise and coordinate clinical, neuroimaging and genetic assessment of tinnitus patients and to aggregate data in a large-scale database in order to identify tinnitus subtypes and their neurobiological underpinnings. The genetics group (WG4) of TINNET is working towards determining the genetic basis of tinnitus subtypes using next generation sequencing technologies and advanced tinnitus phenotyping strategies that will circumvent tinnitus heterogeneity and help to define tinnitus endophenotypes for genetic studies. This will facilitate the development of new therapies and improve the efficacy of currently available treatments ${ }^{67}$. The research aiming at verifying the coincidence of tinnitus and vertigo with genetic predisposition to epilepsy opens the possibility of preventing people from being exposed to environmental factors triggering tinnitus, such as noise 
or ototoxic drugs. In the case of vertigo, this would make it possible to eliminate or thoroughly monitor candidates or professionals whose performance requires outstanding sense of balance.

Additionally, this knowledge could help conduct genetic testing and assist in clinical decision making. Genetic confirmation of the epileptic etiology of tinnitus and vertigo will enable the implementation of anti-epileptic treatments for tinnitus and vestibular disturbances, which is currently not a standard.

\section{Acknowledgements}

Article financed from project INNOSENSE (contract no. STRATEGMED1/248664/7/NCBR/2014). Project co-financed from the funds of National Centre for Research and Development within the framework of STRATEGMED programme.

\section{References}

1 Fransen E, Verstreken M, Verhagen WI, et al. High prevalence of symptoms of Meniere's disease in three families with a mutation in the COCH gene. Hum Mol Genet 1999;8:1425-9.

2 Ding Y, Leng J, Fan F, et al. The role of mitochondrial DNA mutations in hearing loss. Biochem Genet 2013;51:588-602.

3 Fransen E, Verstreken M, Bom SJ, et al. A common ancestor for COCH related cochleovestibular (DFNA9) patients in Belgium and the Netherlands bearing the P51S mutation. J Med Genet 2001;38:61-5.

4 Robertson NG, Resendes BL, Lin JS, et al. Inner ear localization of $m R N A$ and protein products of $\mathrm{COCH}$, mutated in the sensorineural deafness and vestibular disorder, DFNA9. Hum Mol Genet 2001;10:2493-500.

5 Chen DY, Chai YC, Yang T, et al. Clinical characterization of a novel COCH mutation G87V in a Chinese DFNA9 family. Int J Pediatr Otorhinolaryngol 2013;77:1711-5.

6 Yao J, Py BF, Zhu H, et al. Role of protein misfolding in DFNA9 hearing loss. J Biol Chem 2010;285:14909-19.

7 Robertson NG, Lu L, Heller S, et al. Mutations in a novel cochlear gene cause DFNA9, a human nonsyndromic deafness with vestibular dysfunction. Nat Genet 1998;20:299-303.

8 Usami S, Takahashi K, Yuge I, et al. Mutations in the $\mathrm{COCH}$ gene are a frequent cause of autosomal dominant progressive cochleo-vestibular dysfunction, but not of Meniere's disease. Eur J Hum Genet 2003;11:744-8.

9 de Kok YJ, Bom SJ, Brunt TM, et al. A Pro51Ser mutation in the COCH gene is associated with late onset autosomal dominant progressive sensorineural hearing loss with vestibular defects. Hum Mol Genet 1999;8:361-6.

10 Kamarinos M, McGill J, Lynch M, et al. Identification of a novel COCH mutation, I109N, highlights the similar clinical features observed in DFNA9 families. Hum Mutat 2001; 17:351.
11 Collin RW, Pauw RJ, Schoots J, et al. Identification of a novel COCH mutation, G87W, causing autosomal dominant hearing impairment (DFNA9). Am J Med Genet 2006;140:1791-4.

12 Street VA, Kallman JC, Robertson NG, et al. A novel DFNA9 mutation in the $v W F A 2$ domain of $\mathrm{COCH}$ alters a conserved cysteine residue and intrachain disulfide bond formation resulting in progressive hearing loss and site-specific vestibular and central oculomotor dysfunction. Am J Med Genet 2005;139A:86-95.

13 Gallant E, Francey L, Fetting H, et al. Novel COCH mutation in a family with autosomal dominant late onset sensorineural hearing impairment and tinnitus. Am J Otolaryngol 2013;34:230-5.

14 Makishima T, Rodriguez CI, Robertson NG, et al. Targeted disruption of mouse Coch provides functional evidence that DFNA9 hearing loss is not a COCH haploinsufficiency disorder. Hum Genet 2005;118:29-34.

15 Bae SH, Robertson NG, Cho HJ, et al. Identification of pathogenic mechanisms of COCH mutations, abolished cochlin secretion, and intracellular aggregate formation: genotypephenotype correlations in DFNA9 deafness and vestibular disorder. Hum Mutat 2014;35:1506-13.

16 Kim BJ, Kim AR, Han KH, et al. Distinct vestibular phenotypes in DFNA9 families with COCH variants. Eur Arch Otorhinolaryngol 2016;273:2993-3002.

17 Wingard JC, Zhao HB. Cellular and deafness mechanisms underlying connexin mutation-induced hearing loss - a common hereditary deafness. Front Cell Neurosci 2015;9:202.

18 Tsukada K, Nishio SY, Hattori M, et al. Ethnic-specific spectrum of GJB2 and SLC26A4 mutations: their origin and a literature review. Ann Otol Rhinol Laryngol 2015;124(Suppl 1):61S-76S.

19 Zheng J, Ying Z, Cai Z, et al. GJB2 mutation spectrum and genotype-phenotype correlation in 1067 Han Chinese subjects with non-syndromic hearing loss. PLoS One 2015;10:e0128691.

20 del Castillo I, Villamar M, Moreno-Pelayo MA, et al. A deletion involving the connexin 30 gene in nonsyndromic hearing impairment. N Engl J Med 2002;346:243-9.

21 Del Castillo I, Moreno-Pelayo MA, Del Castillo FJ, et al. Prevalence and evolutionary origins of the del(GJB6D13S1830) mutation in the DFNB1 locus in hearingimpaired subjects: a multicenter study. Am J Hum Genet 2003;73:1452-8.

22 Seeman P, Bendova O, Raskova D, et al. Double heterozygosity with mutations involving both the GJB2 and GJB6 genes is a possible, but very rare, cause of congenital deafness in the Czech population. Ann Hum Genet 2005;69:9-14.

23 Kabahuma RI, Ouyang X, Du LL, et al. Absence of GJB2 gene mutations, the GJB6 deletion (GJB6-D13S1830) and four common mitochondrial mutations in nonsyndromic genetic hearing loss in a South African population. Int J Pediatr Otorhinolaryngol 2011;75:611-7.

24 Lasisi AO, Bademci G, Foster J, et al. Common genes for non-syndromic deafness are uncommon in sub-Saharan Af- 
rica: a report from Nigeria. Int $\mathrm{J}$ Pediatr Otorhinolaryngol 2014;78:1870-3.

25 Wonkam A, Bosch J, Noubiap JJ, et al. No evidence for clinical utility in investigating the connexin genes GJB2, GJB6 and GJA1 in non-syndromic hearing loss in black Africans. S Afr Med J 2015;105:23-6.

26 Bosch J, Noubiap JJ, Dandara C, et al. Sequencing of GJB2 in Cameroonians and Black South Africans and comparison to 1000 genomes project data support need to revise strategy for discovery of nonsyndromic deafness genes in Africans. OMICS 2014;18:705-10.

27 Busi M, Castiglione A, Taddei Masieri M, et al. Novel mutations in the SLC26A4 gene. Int J Pediatr Otorhinolaryngol 2012;76:1249-54

28 Miyagawa M, Nishio SY, Usami S. Mutation spectrum and genotype-phenotype correlation of hearing loss patients caused by SLC26A4 mutations in the Japanese: a large cohort study. J Hum Genet 2014;59:262-8.

29 Frejo L, Giegling I, Teggi R, et al. Genetics of vestibular disorders: pathophysiological insights. J Neurol 2016;263(Suppl 1):S45-53.

30 Schrauwen I, Hasin-Brumshtein Y, Corneveaux JJ, et al. A comprehensive catalogue of the coding and non-coding transcripts of the human inner ear. Hear Res 2016;333:266-74.

31 Li L, Wang Y, An L, et al. A network-based method using a random walk with restart algorithm and screening tests to identify novel genes associated with Meniere's disease. PLoS One 2017;12:e182592.

32 Eppsteiner RW, Smith RJ. Genetic disorders of the vestibular system. Curr Opin Otolaryngol Head Neck Surg 2011;19:397-402.

33 Teggi R, Zagato L, Delli Carpini S, et al. Genetics of ion homeostasis in Meniere's Disease. Eur Arch Otorhinolaryngol 2017;274:757-63.

34 Tsai KL, Wang CT, Kuo CH, et al. The potential role of epigenetic modulations in BPPV maneuver exercises. Oncotarget 2016;7:35522-34.

35 Requena T, Espinosa-Sanchez JM, Lopez-Escamez JA. Genetics of dizziness: cerebellar and vestibular disorders. Curr Opin Neurol 2014;27:98-104.

36 Gazquez I, Lopez-Escamez JA. Genetics of recurrent vertigo and vestibular disorders. Curr Genomics 2011;12:443-50.

37 Lee H, Jen JC, Wang H, et al. A genome-wide linkage scan of familial benign recurrent vertigo: linkage to $22 q 12$ with evidence of heterogeneity. Hum Mol Genet 15 2006;15:251-8.

38 Lopez-Escamez JA, Bibas T, Cima RF, et al. Genetics of Tinnitus: an emerging area for molecular diagnosis and drug development. Front Neurosci 2016;10:377.

39 Sand PG, Langguth B, Kleinjung T. Deep resequencing of the voltage-gated potassium channel subunit KCNE3 gene in chronic tinnitus. Behav Brain Funct 2011;7:39.

40 Sand PG, Luettich A, Kleinjung T, et al. An examination of KCNE1 mutations and common variants in chronic Tinnitus. Genes (Basel) 2010;1:23-37.
41 Pawelczyk M, Rajkowska E, Kotylo P, et al. Analysis of inner ear potassium recycling genes as potential factors associated with tinnitus. Int J Occup Med Environ Health 2012;25:356-64.

42 Vetter DE, Mann JR, Wangemann P, et al. Inner ear defects induced by null mutation of the isk gene. Neuron 1996; 17:1251-64.

43 Letts VA, Valenzuela A, Dunbar C, et al. A new spontaneous mouse mutation in the Kcnel gene. Mamm Genome 2000;11:831-5.

44 Yang T, Gurrola JG, 2nd, Wu H, et al. Mutations of KCNJ10 together with mutations of SLC26A4 cause digenic nonsyndromic hearing loss associated with enlarged vestibular aqueduct syndrome. Am J Hum Genet 2009;84:651-7.

45 Kubisch C, Schroeder BC, Friedrich T, et al. KCNQ4, a novel potassium channel expressed in sensory outer hair cells, is mutated in dominant deafness. Cell 1999;96:437-46.

46 Coucke PJ, Van Hauwe P, Kelley PM, et al. Mutations in the KCNQ4 gene are responsible for autosomal dominant deafness in four DFNA2 families. Hum Mol Genet 1999;8:1321-8.

47 Uehara DT, Freitas EL, Alves LU, et al. A novel KCNQ4 mutation and a private IMMP2L-DOCK4 duplication segregating with nonsyndromic hearing loss in a Brazilian family. Hum Genome Var 2015;2:15038.

48 Kim HJ, Lv P, Sihn CR, et al. Cellular and molecular mechanisms of autosomal dominant form of progressive hearing loss, DFNA2. J Biol Chem 2011;286:1517-27.

49 Szczepaniak WS, Moller AR. Effects of (-)-baclofen, clonazepam, and diazepam on tone exposure-induced hyperexcitability of the inferior colliculus in the rat: possible therapeutic implications for pharmacological management of tinnitus and hyperacusis. Hear Res 1996;97:46-53.

50 Schwenk J, Metz M, Zolles G, et al. Native $G A B A(B)$ receptors are heteromultimers with a family of auxiliary subunits. Nature 2010;465:231-5.

51 Sand PG, Langguth B, Itzhacki J, et al. Resequencing of the auxiliary $G A B A(B)$ receptor subunit gene KCTD12 in chronic tinnitus. Front Syst Neurosci 2012;6:41.

52 Sand PG, Langguth B, Kleinjung T, et al. Genetics of chronic tinnitus. Prog Brain Res 2007;166:159-68.

53 Sand P, Langguth B, Schecklmann M, et al. GDNF and BD$N F$ gene interplay in chronic tinnitus. Int J Mol Epidemiol Genet 2012;3:245-51.

54 Weisz N, Hartmann T, Muller N, et al. Alpha rhythms in audition: cognitive and clinical perspectives. Front Psychol 2011;2:73.

55 Pawlak-Osinska K, Kazmierczak W, Kazmierczak H, et al. Cortical activity in tinnitus patients and its modification by phonostimulation. Clinics (Sao Paulo) 2013;68:511-5.

56 Poduri A, Lowenstein D. Epilepsy genetics past, present, and future. Curr Opin Genet Dev 2011;21:325-32.

57 Helbig I, Scheffer IE, Mulley JC, et al. Navigating the channels and beyond: unravelling the genetics of the epilepsies. Lancet Neurol 2008;7:231-45.

58 Steffens M, Leu C, Ruppert AK, et al. Genome-wide asso- 
ciation analysis of genetic generalized epilepsies implicates susceptibility loci at 1q43, 2p16.1, 2q22.3 and 17q21.32. Hum Mol Genet 2012;21:5359-72.

59 Epilepsies ILAECoC. Genetic determinants of common epilepsies: a meta-analysis of genome-wide association studies. Lancet Neurol 2014;13:893-903.

${ }^{60} \mathrm{Li} \mathrm{S}$, Choi V, Tzounopoulos T. Pathogenic plasticity of Kv7.2/3 channel activity is essential for the induction of tinnitus. Proc Natl Acad Sci USA 2013;110:9980-5.

${ }^{61}$ Kalappa BI, Soh H, Duignan KM, et al. Potent KCNQ2/3specific channel activator suppresses in vivo epileptic activity and prevents the development of tinnitus. J Neurosci 2015;35:8829-42.

62 Hewett R, Bartolomei F. Epilepsy and the cortical vestibular system: tales of dizziness and recent concepts. Front Integr Neurosci 2013;7:73.
63 Tarnutzer AA, Lee SH, Robinson KA, et al. Clinical and electrographic findings in epileptic vertigo and dizziness: a systematic review. Neurology 2015;84:1595-604.

64 Pawlak-Osinska K, Kazmierczak H, Kuczynska R, et al. Vestibular findings in children's epilepsy. Otolaryngol Pol 1999;53:479-83.

65 Monzani D, Genovese E, Pini LA, et al. Nimodipine in otolaryngology: from past evidence to clinical perspectives. Acta Otorhinolaryngol Ital 2015;35:135-45.

66 Furman JM, Marcus DA, Balaban CD. Vestibular migraine: clinical aspects and pathophysiology. Lancet Neurol 2013;12:706-15.

67 Cederroth CR, Lopez-Escamez JA. Genetics WG4. Improving diagnosis and drug development through the genetic understanding of tinnitus subtypes: a TINNET endeavour. ENT \& Audiology News 2016;24:87-8.

Received: March 23, 2017 - Accepted: October 12, 2017

Address for correspondence: Tomasz Grzybowski, Department of Forensic Medicine, Division of Molecular and Forensic Genetics, Ludwik Rydygier Collegium Medicum Nicolaus Copernicus University, Skłodowskiej-Curie 9, 85-094 Bydgoszcz, Poland. Tel. +48 52585 3886. Fax +48 52585 3553. E-mail: tgrzyb@cm.umk.pl 